\title{
Expression and Prognostic Value of Tumor-Infiltrating Lymphocytes and PD-LI in Hepatocellular Carcinoma
}

This article was published in the following Dove Press journal: OncoTargets and Therapy

\author{
Hanxiao Nie \\ Tao He \\ Li Wang \\ Ling Zhang
}

Department of Hepatobiliary Surgery, The Affiliated Cancer Hospital of Zhengzhou University, Zhengzhou, 450008, People's Republic of China

Correspondence: Hanxiao Nie Department of Hepatobiliary Surgery The Affiliated Cancer Hospital of

Zhengzhou University, 127 Dongming Road, Jinshui District, Zhengzhou, 450008, People's Republic of China Tel +861529005586I

Emailnie9II6532I3@I63.com

Ling Zhang

Department of Hepatobiliary Surgery,

The Affiliated Cancer Hospital of

Zhengzhou University, 127 Dongming

Road, Jinshui District, Zhengzhou,

450008, People's Republic of China

$\mathrm{Tel} / \mathrm{Fax}+8637 \mathrm{I} 65587787$

Email zhanglily56I@I26.com
Aim: To explore the difference in tumor-infiltrating lymphocytes (TILs) and programmed death-ligand (PD-L1) in primary hepatocellular carcinoma (HCC) and its adjacent tissues, and to evaluate their effect on HCC prognosis.

Methods: Liver cancer and paracancerous tissue samples were collected from 72 patients who underwent radical hepatectomy between December 15, 2017 and January 9, 2019. Flow cytometry was used to detect the distribution of TILs and PD-L1, analyze the correlation between the expression of CD8/CD3 and PD-L1 and clinical-pathological parameters, and evaluate their effect on the prognosis of HCC patients.

Results: The distribution proportion of CD3+T cells, CD4+T cells, and PD-L1 in liver cancer were significantly higher than in paracancerous tissues, while the distribution proportion of $\mathrm{CD} 8+\mathrm{T}$ cells was significantly lower (all $\mathrm{P}<0.05$ ). In $\mathrm{HCC}$, the distribution proportion of CD8+T cells was related to tumor size and stage, while the PD-L1 expression was related to the tumor stage only (all $\mathrm{P}<0.05$ ). Univariate analysis showed that tumor differentiation, TNM stage, expression of CD8/CD3, and PD-L1 in tumor tissue were related to disease-free survival $(\mathrm{DFS})(\mathrm{P}<0.05)$; multivariate Cox regression analysis showed that tumor differentiation, TNM stage, CD8/CD3, and PD-L1 expression were independent influencing factors of postoperative DFS $(\mathrm{P}<0.05)$. Kaplan-Meier survival curve analysis showed that the DFS of CD8/CD3 high expression group was significantly higher than that of the low expression group, and the DFS of PD-L1 low expression group was significantly higher than that of the high expression group (all $\mathrm{P}<0.05$ ).

Conclusion: There are significant differences in the distribution of TILs and PD-L1 in HCC and paracancerous tissues. The expression of CD8/CD3 and PD-L1 in tumor-infiltrating lymphocytes in HCC may help evaluate the immunological indexes of prognosis after radical resection of HCC and to further the study of immunotherapy in patients with HCC.

Keywords: liver cancer, TILs, PD-L1, DFS

\section{Introduction}

Hepatocellular carcinoma (HCC) is one of the most common malignant tumors worldwide, with high morbidity and mortality and poor prognosis. At present, surgical treatment is still considered as the most effective treatment for liver cancer. Early diagnosis and early treatment of liver cancer are essential for the radical operation of liver cancer. Yet, most cases present with a middle and late stage.

Over the last decade, biotherapy has gradually become a new and fundamental treatment strategy for patients with advanced liver cancer. The immune 
microenvironment has an important role in cellular immunotherapy. Tumor-infiltrating lymphocytes (TILs) are essential parts of the cellular immune microenvironment. In general, the vast majority of TILs are CD3+T cells, which can be divided into two subgroups: CD4+T cells and CD8+T cells.Moreover, programmed death factor-1 (PD-1) is another checkpoint molecule in the immune response. PD-L1, a ligand of PD-1, is expressed in a variety of tumor cells. The combination of PD-L1 and PD-1 can inhibit the proliferation of TILs. Nowadays, a number of studies have reported that TILs and PD-L1 are closely related to the prognosis and survival of tumor patients. ${ }^{2-5}$ The purpose of this study was to detect the distribution ratio of TILs and PD-L1 in liver cancer tissues and adjacent tissues by flow cytometry so as to understand the changes of the immune microenvironment in patients with liver cancer, and to analyze the correlation between the expression of PD-L1 and TILs and clinical-pathological parameters and postoperative prognosis. Moreover, this study explored the possibility of using PD-L1 and TILs as biomarkers for the prognosis and survival of patients with liver cancer.

\section{Materials and Methods}

\section{Research Object}

Liver tissue samples were collected from 72 patients who underwent radical hepatectomy at the Department of Hepatobiliary Surgery, affiliated with Cancer Hospital of Zhengzhou University between December 2017 and January 2019. All patients had complete clinicopathological data. There were 51 males and 21 females patients aged 30 to 84 years old, with an average age of (54.1 \pm 10.7 ) years. All patients were diagnosed with hepatitis B associated hepatocellular carcinoma. They all had a history of hepatitis B virus (HBV) infection; serum HBsAg or HBV-DNA were positive before the operation. Hepatocellular carcinoma was confirmed by pathology after the operation.

No patients received other treatments, such as radiotherapy, chemotherapy, and immunotherapy, before the operation. The tumor was completely removed during the operation. Anatomical hepatectomy or the cutting edge was more than $2 \mathrm{~cm}$ from the tumor, and the postoperative pathological examination was negative.

The hospital ethics committee approved the study, and informed consent was signed by patients.(The ethics committee of The Affiliated Cancer Hospital of Zhengzhou University, The ethical code is 2016CT054).

\section{Detection of Infiltrating Lymphocytes in Liver Tissue by Flow Cytometry} Main Reagents and Instruments

FITC anti-human CD4 antibody PE/CY7 anti-human CD3 antibody, FITC anti-human CD8 antibody, APC antihuman PD-L1 antibody, FITC anti-human CD4 antibody, APC Mouse IgG1, $\kappa$ Isotype Ctrl (FC) antibody, and 7AAD active staining solution were obtained from American Biolegend company. RPMI1640 was purchased from the American Gibco company. FACSCanto II flow cytometry was obtained from American BD company.

\section{Preparation of Single-Cell Suspension from Fresh Liver Cancer and Paracancerous Specimens}

The resected specimens were stored in RPMI1640 culture medium at $4{ }^{\circ} \mathrm{C}$ immediately upon collection. After the necrotic tissue was removed by scissors, the liver cancer and paracancerous specimens were cut into small pieces. The specimens were then grinded by manual tissue grinder until the tissue blocks were not visible to the naked eye, filtered suing $70 \mu \mathrm{m}$ filter, and centrifuged at $300 \mathrm{~g}$ for $5 \mathrm{~min}$. Finally, the cells were collected for flow staining.

\section{Flow Cytometry}

A total of $1 \times 10^{7} \mathrm{~mL} /$ tube (100ul/tube) mixed with PBS were added to three flow tubes (labeled tube 1, 2, 3). Tube 1 was then mixed with the following reagents: $5 \mathrm{ul}$ FITC anti-human CD8, 5ul PE/CY7 anti-human CD3, and 5ul APC Mouse IgG1; tube 2 with 5ul FITC antihuman CD8, 5ul PE/CY7anti-human CD3, and 5ul APC anti-human PD-L1; and tube 3 with 5ul FITC anti-human CD4, 5ul PE/CY7 anti-human CD3, and 5ul APC antihuman PD-L1.

After incubation at room temperature for 20 minutes in dark, samples were washed with PBS for two times, mixed with 200ul PBS resuspension cells and 10ul of 7AAD active staining agent for 10 minutes. Consequently, samples were analyzed using flow cytometry.

\section{Follow-Up}

All patients were regularly followed up according to reexamination (serum tumor marker alpha-fetoprotein (AFP) and abdominal B-mode ultrasound) and telephone contact 
until the death or the end of the study. Tumor recurrence was the primary endpoint of this study. The disease-free survival (DFS) was the main observation index defined as the time from the date of complete resection of the tumor to the time of recurrence or metastasis or death due to disease progression. The diagnosis of tumor recurrence or metastasis was based on enhanced CT or magnetic resonance imaging and serum AFP levels. The follow-up was performed from December 2017 to June 2020.

\section{Statistical Analysis}

The experimental data were analyzed by SPSS22.0 statistical software. The data of continuous variables were expressed by $(\mathrm{x} \pm \mathrm{s})$, and the differences between paired samples (liver cancer tissues and paracancerous tissues) were tested by Nonparametric Test. The cut-off values of CD8/CD3 and PD-L1 were determined according to the receiver operating zone line (ROC). The cut-off values are 42.6 and 5.8. According to this cut-off value, CD8/CD3 and PD-L1 were divided into high expression groups and low expression groups. Kaplan-Meier method was used to analyze the survival time of the two groups, and the Logrank method was used to test the survival rate. Univariate and multivariate Cox regression analysis was used to analyze the independent prognostic factors. The difference was statistically significant $(\mathrm{P}<0.05)$.

\section{Results}

Flow Analysis of Infiltrating Lymphocytes and PD-LI in Hepatocellular Carcinoma and Paracancerous Tissues (Figures I and 2) Differences in the Distribution Proportion of Primary Anti-Tumor Effector Cells in Hepatocellular Carcinoma and Paracancerous Tissues

The flow test analysis indicated that the proportion of CD3 +T cells and CD4+T cells in the hepatocellular carcinoma group was significantly higher than that in the paracancerous group $(\mathrm{P}=0.002, \mathrm{P}<0.001)$. Moreover, the proportion of $\mathrm{CD} 8+\mathrm{T}$ cells in the hepatocellular carcinoma group was significantly lower than that in the paracancerous group ( $\mathrm{P}$ $<0.001)$. The ratio of $\mathrm{CD} 4+\mathrm{T}$ cells to $\mathrm{CD} 8+\mathrm{T}$ cells in the HCC group was significantly higher than that in the paracancerous group $(\mathrm{P}<0.001)$, which was out of balance. The distribution proportion of PD-L1 protein in the hepatocellular carcinoma group was significantly higher than that in the paracancerous group $(\mathrm{P}<0.001)$ (Table 1).
The Relationship Between the Proportion of CD8/CD3, the Expression of PD-LI, and Clinicopathological Factors in Hepatocellular Carcinoma

The above results showed that the expression of CD8/CD3 in HCC tissues was significantly lower than in paracancerous tissues, while the expression of PD- was significantly higher. Therefore, we further analyzed the relationship between the expression of CD8/CD3 and PD-L1 in HCC and the patient's age, sex, tumor size, degree of differentiation, TNM stage, liver cirrhosis, and preoperative AFP level. The results showed that the expression of CD8/CD3 in $\mathrm{HCC}$ was related to tumor size and TNM stage. In contrast, the expression of PD-L1 was related to the TNM stage and had no significant correlation with other clinical indexes, as shown in Table 2.

The CD8/CD3 in Liver Cancer Tissue is the Influencing Factor of (DFS) in Postoperative Disease-Free Survival of Patients with Liver Cancer

Factors affecting the postoperative disease-free survival in patients with liver cancer were further analyzed. Univariate Cox regression analysis showed that tumor differentiation, TNM stage, expression of $\mathrm{CD} 8 / \mathrm{CD} 3$, and PD-L1 in tumor tissue were related to postoperative DFS. Multivariate Cox regression analysis showed that the degree of tumor differentiation and the expression of CD8/CD3 and PD-L1 were independent influencing factors of postoperative DFS (Table 3). Moreover, the Kaplan-Meier survival curve analysis showed that the DFS of CD8/CD3 high expression group was significantly higher than that of the low expression group, and the DFS of PD-L1 low expression group was significantly higher than that of the high expression group (Figure 3).

\section{Discussion}

Surgical resection, transcatheter arterial chemoembolization, radiofrequency ablation, and tyrosine kinase inhibitors (such as sorafenib) are the primary treatment methods for patients with HCC. Yet, the overall prognosis of liver cancer is still not satisfactory. ${ }^{1,6,7}$

Immunotherapy has an important role in the treatment of malignant tumors. It can reduce the tumor's load and prevent other chronic liver lesions from developing into HCC. ${ }^{8,9}$ TILs are the type of cells that participate in the 

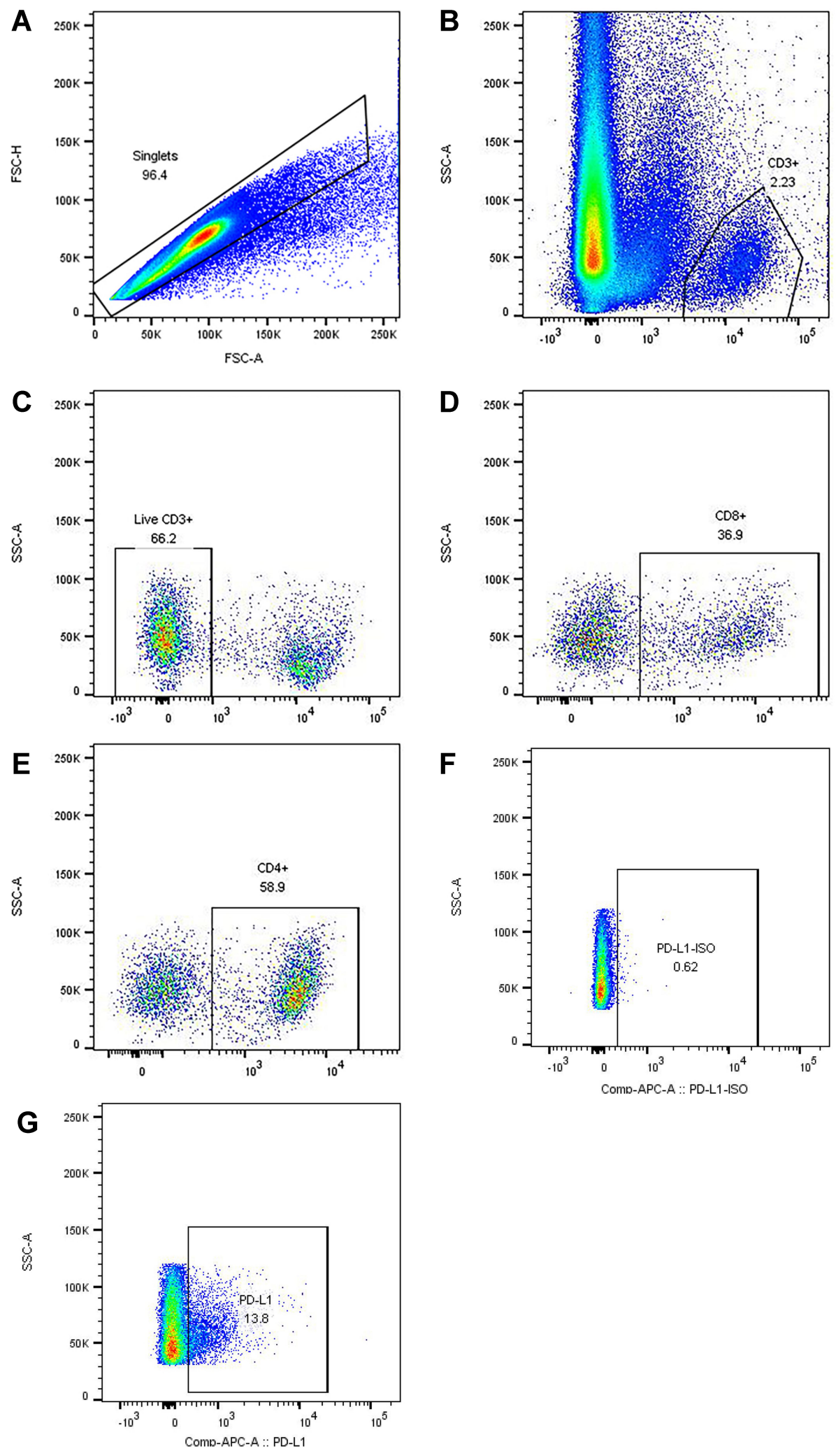

Figure I Flow diagram of tumor infiltrating lymphocytes and PD-LI in hepatocellular carcinoma. (A) Removal of adhesion of cells by forward angular scattering of FSC-An and FSH-H. (B) CD3 sets up a door to circle out CD3+T cells. (C) Elimination of dead cells by setting a gate through 7AAD. (D and E) Set up a gate to circle the CD4+ cells and $\mathrm{CD} 8+$ cells in CD3+T cells. ( $\mathbf{F}$ and $\mathbf{G})$ Through the control tube of the same type, the PD-LI positive group was circled. 

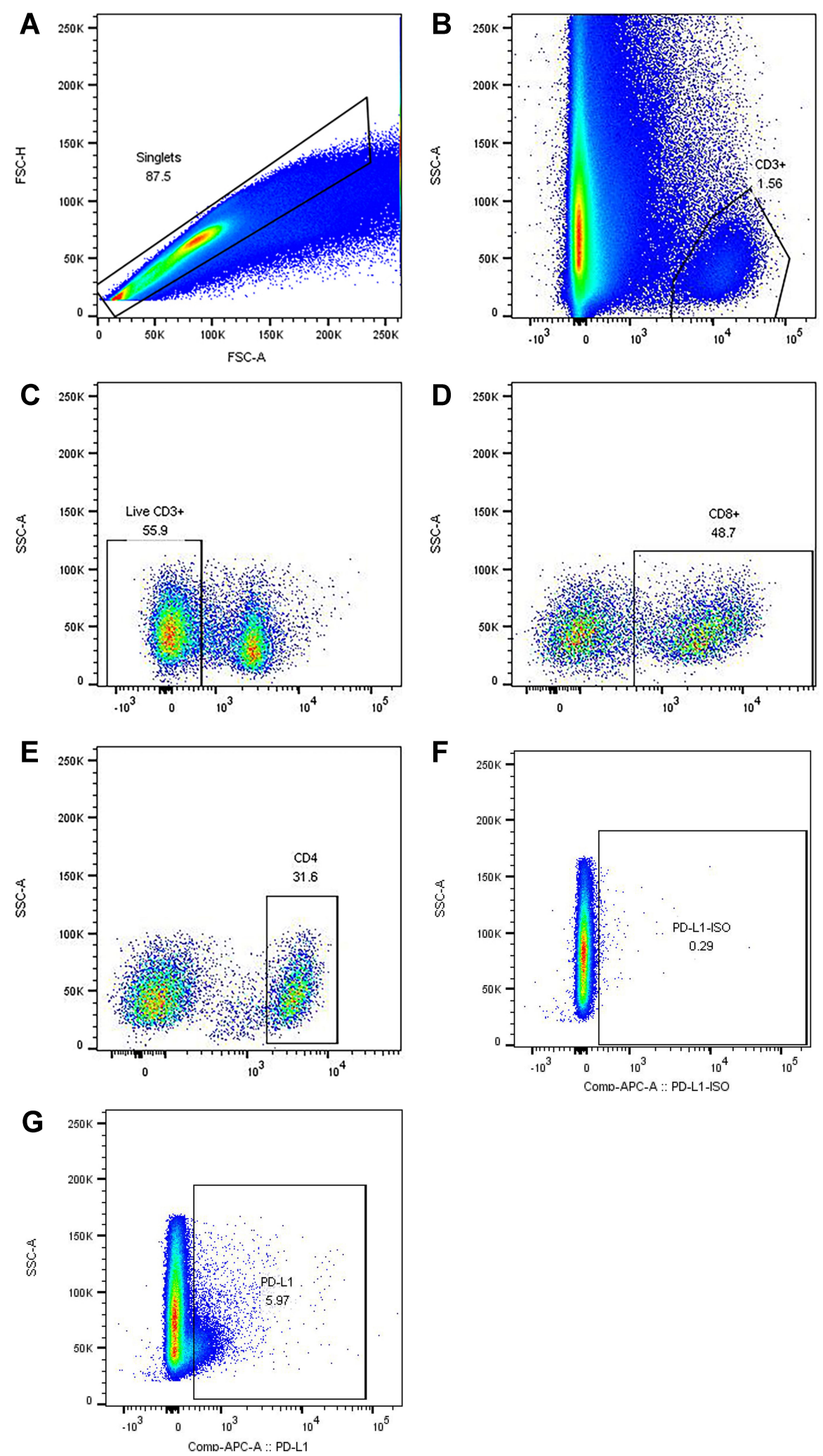

Figure 2 Flow diagram of tumor infiltrating lymphocytes and PD-LI in paracancerous tissues. (A) Removal of adhesion of cells by forward angular scattering of FSC-An and FSH-H. (B) CD3 sets up a door to circle out CD3+T cells. (C) Elimination of dead cells by setting a gate through 7AAD. (D and E) Set up a gate to circle the CD4+ cells and CD8+ cells in CD3+T cells. ( $F$ and $\mathbf{G})$ Through the control tube of the same type, the PD-LI positive group was circled. 
Table I Distribution Proportion of Main Anti-Tumor Effector Cells and PD-LI in Hepatocellular Carcinoma and Paracancerous Tissues

\begin{tabular}{|l|c|c|c|}
\hline & Cancer & Adjacent to Cancer & P \\
\hline $\mathrm{CD}^{+}$ & $4.53(2.06,10.50)$ & $3.14(1.89,5.59)$ & 0.026 \\
\hline $\mathrm{CD}^{+} / \mathrm{CD}^{+}$ & $51.30(32.80,62.10)$ & $66.20(56.45,71.50)$ & $<0.001$ \\
\hline $\mathrm{CD}^{+} / \mathrm{CD}^{+}$ & $45.10(33.70,61.05)$ & $24.00(20.00,30.70)$ & $<0.001$ \\
\hline $\mathrm{CD}^{+} / \mathrm{CD}^{+}$ & $0.88(0.53,1.86)$ & $0.36(0.28,0.53)$ & $<0.001$ \\
\hline $\mathrm{PD}-\mathrm{LI}$ & $9.18(4.91,15.85)$ & $6.46(2.90,9.90)$ & 0.01 \\
\hline
\end{tabular}

body's anti-tumor immune response and have a specific anti-tumor effect. ${ }^{10}$ Among them, $\mathrm{T}$ cells are the most important tumor-infiltrating lymphocytes. In general, the vast majority of TILs are CD3+T cells, which can be divided into two subgroups: $\mathrm{CD} 4+\mathrm{T}$ cells and $\mathrm{CD} 8+\mathrm{T}$ cells. ${ }^{11} \mathrm{CD} 8+\mathrm{T}$ cells are the most important effector cells in the process of the anti-tumor immune response. CD4+T lymphocytes mainly refer to helper $\mathrm{T}$ cells, which can

Table 2 Relationship Between the Expression of CD8/CD3 and PD-LI in Hepatocellular Carcinoma and Clinicopathological Factors

\begin{tabular}{|c|c|c|c|c|c|c|c|}
\hline Characteristics & Cases & CD8/CD3 & $\mathbf{t}$ & $\mathbf{P}$ & PD-LI & $\mathbf{t}$ & $\mathbf{P}$ \\
\hline Age (years) & & & 0.266 & 0.791 & & 0.769 & 0.444 \\
\hline$<60$ & 51 & $49.2 \pm 27.90$ & & & $11.39 \pm 8.99$ & & \\
\hline$\geq 60$ & 21 & $48.03 \pm 14.32$ & & & $13.40 \pm 12.47$ & & \\
\hline Gender & & & 0.934 & 0.354 & & 0.585 & 0.561 \\
\hline Male & 51 & $47.66 \pm 17.63$ & & & $12.42 \pm 10.82$ & & \\
\hline Female & 21 & $51.75 \pm 14.77$ & & & $10.89 \pm 8.15$ & & \\
\hline Tumor size $(\mathrm{cm})$ & & & 3.231 & 0.002 & & 1.211 & 0.230 \\
\hline$<5$ & 23 & $57.65 \pm 14.85$ & & & $14.07 \pm 10.48$ & & \\
\hline$\geq 5$ & 49 & $44.73 \pm 16.25$ & & & $10.99 \pm 9.85$ & & \\
\hline Differentiation & & & 0.649 & 0.519 & & 0.045 & 0.964 \\
\hline High and medium & 46 & $48.14 \pm 17.27$ & & & $11.95 \pm 10.52$ & & \\
\hline Low & 26 & $51.18 \pm 15.65$ & & & $12.07 \pm 8.82$ & & \\
\hline TNM stage & & & 2.493 & 0.015 & & 2.229 & 0.029 \\
\hline I, II & 37 & $53.50 \pm 15.95$ & & & $9.47 \pm 6.67$ & & \\
\hline III, IV & 35 & $43.95 \pm 16.57$ & & & $14.63 \pm 12.29$ & & \\
\hline Cirrhosis & & & 0.420 & 0.676 & & 1.689 & 0.096 \\
\hline No & 12 & $50.73 \pm 17.65$ & & & $7.54 \pm 2.77$ & & \\
\hline Yes & 60 & $48.48 \pm|6.8|$ & & & $12.86 \pm 10.77$ & & \\
\hline $\operatorname{AFP}(\mu g / L)$ & & & 0.857 & 0.394 & & 0.178 & 0.860 \\
\hline$<20$ & 15 & $45.53 \pm 17.92$ & & & $11.56 \pm 10.25$ & & \\
\hline$\geq 20$ & 57 & $49.73 \pm 16.60$ & & & $12.08 \pm 10.13$ & & \\
\hline
\end{tabular}


Table 3 Univariate and Multivariate Cox Regression Analysis of Postoperative Prognosis in 72 Patients with Hepatocellular Carcinoma

\begin{tabular}{|l|l|l|l|l|}
\hline \multirow{2}{*}{ Variable } & \multicolumn{2}{l|}{ Univariate Analysis } & \multicolumn{2}{l|}{ Multifactor Analysis } \\
\cline { 2 - 4 } & HR(95\% CI) & $\mathbf{P}$ & HR(95\% CI) \\
\hline Age (years) & $0.538(0.208 \sim 1.387)$ & 0.200 & \\
\hline Gender(Male/Female) & $1.132(0.520 \sim 2.464)$ & 0.755 & \\
\hline Tumor size (cm) & $0.510(0.230 \sim 1.132)$ & 0.098 & & \\
\hline Differentiation(High and medium/Low & $5.152(2.393 \sim 11.095)$ & $<0.001$ & $4.720(1.962 \sim 11.355)$ \\
\hline TNM stage(I II/III IV) & $3.539(1.667 \sim 7.514)$ & 0.001 & & $<0.001$ \\
\hline Cirrhosis (Yes/No) & $1.372(0.487 \sim 3.869)$ & 0.549 & & \\
\hline AFP ( $\mu$ g/L) & $2.064(0.747 \sim 5.702)$ & 0.162 & & \\
\hline CD8/CD3(Higher/Lower expression) & $0.087(0.036 \sim 0.212)$ & 0.001 & $0.083(0.032 \sim 0.211)$ \\
\hline PD-LI (Higher/Lower expression) & $0.410(0.192 \sim 0.876)$ & 0.021 & $0.440(0.202 \sim 0.959)$ \\
\hline
\end{tabular}

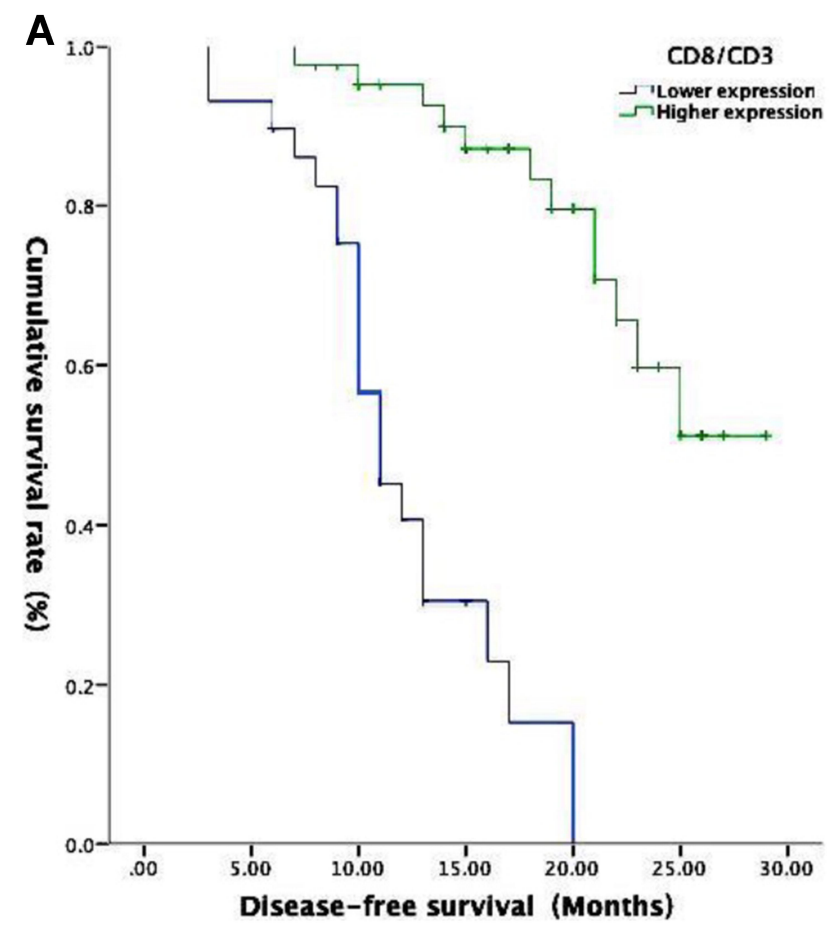

Figure 3 Kaplan-Meier survival analysis curve. (A) CD8/CD3; (B) PD-LI.

regulate the immune response of other $\mathrm{T}$ cell groups and assist $\mathrm{B}$ cells in secreting antibodies. The $\mathrm{CD} 4+\mathrm{T} / \mathrm{CD} 8+\mathrm{T}$ ratio is crucial for the immune response and immune regulation; its changes reflect the immune state. ${ }^{12,13}$ Some studies have found that lower CD8+TILs levels are related to some clinicopathological parameters, such as negative HBsAg, large tumor size, and late TNM stage. In addition, the high-density CD8+TILs have been related to better OS and DFS. ${ }^{14}$

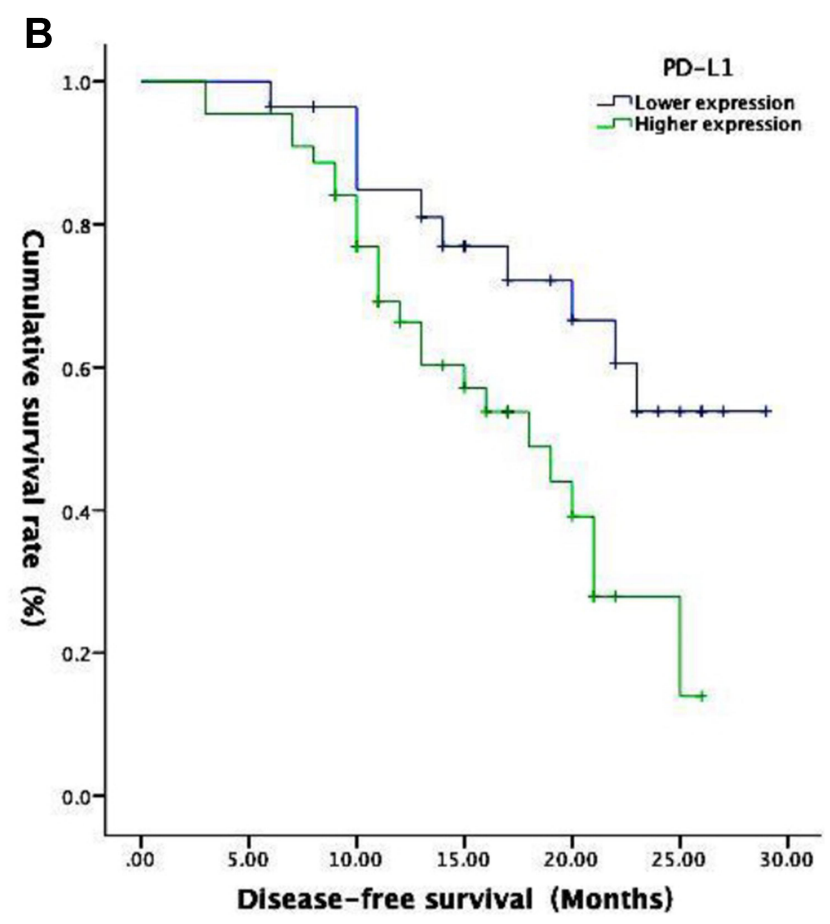

In this study, we found that the expression of CD8/ CD3 in hepatocellular carcinoma is associated with the tumor size and stage. This further suggests that TILs are an important factor in the mechanism of HCC immunosuppression. The body's immune status is related to the invasive activity of TILs, which indicates that the low immunity is one of the important factors of tumor growth, recurrence, and metastasis. Furthermore, our multiple Cox regression analysis 
revealed that $\mathrm{CD} 8 / \mathrm{CD} 3$ is an independent protective factor affecting the postoperative prognosis of patients with liver cancer.

Programmed death factor-1 (PD-1) is a checkpoint molecule in the immune response. By binding with PD1, PD-L1 blocks the secretion, proliferation, and cytotoxic cytokine secretion of $\mathrm{T}$ lymphocytes, resulting in a decrease in T lymphocytes' distribution. ${ }^{3,15}$ At present, the inhibitor of immune checkpoint molecule (PD-L1/PD1) has achieved remarkable results in many malignant tumors. It has been found that its clinical efficacy is closely related to the expression of PD-L1 in tumors. In addition, PD-L1 positive patients, in comparison with PDL1 negative patients, are twice as likely to undergo relapse and have greater numbers of tumors with vascular invasion. Yet, its clinical value in HCC needs further assessment. $^{16,17}$

Our data indicated that the expression of PD-L1 in HCC was associated with the clinical stage. The clinical stage could further affect the prognosis of patients. Multiple Cox regression analysis showed that PD-L1 was an independent factor affecting the postoperative prognosis of patients with hepatocellular carcinoma. DFS in the group with high expression of PD-L1 was significantly lower than that in patients with low expression of PD-L1. Therefore, PD-L1 may also be used as a prognostic biomarker of HCC and to evaluate immune checkpoint inhibitors' clinical efficacy.

This study has a few limitations. First, the study has a small sample size. Second, most of the patients in this study received resection, radiofrequency ablation, interventional therapy, immunotherapy, and other related comprehensive treatments for recurrent or metastatic tumors once they were found to have recurrence or metastasis after the operation. These treatments may have a different effect on the overall survival time (OS) of the patients. Therefore, the overall survival time of the patients was not evaluated in this study. The small sample size is another limiting factor in this study.

To sum up, our data suggest that CD8+T and PD-L1 have independent and significant effects on the survival and prognosis of patients with HCC. Thus, CD8+T and PD-L1 could potentially become prognostic biomarkers for HCC. Future studies should include a larger sample size and should explore the relationship between TILs, PD-L1, and overall survival (OS) further.

\section{Supportive Foundations}

This research did not receive any specific grant from funding agencies in the public, commercial, or not-forprofit sectors.

\section{Institutional Review Board Statement}

This experiment has got the approval of the Medical Ethics Committee of Henan Tumor Hospital Affiliated To Zhengzhou University and this study is in line with the Declaration of Helsinki.

\section{Abbreviations}

HCC, Hepatocellular carcinoma.

\section{Acknowledgments}

This study was supported by the Basic and Frontier Technology Research Program of Henan Province [NO.162300410108].

\section{Disclosure}

The authors report no conflicts of interest in this work.

\section{References}

1. Liu CY, Chen KF, Chen PJ. Treatment of liver cancer. Cold Spring Harb Perspect Med. 2015;5(9):a021535. doi:10.1101/cshperspect. a021535

2. Inarrairaegui M, Melero I, Sangro B. Immunotherapy of hepatocellular carcinoma: facts and hopes. Clin Cancer Res. 2018;24 (7):1518-1524. doi:10.1158/1078-0432.CCR-17-0289

3. Huang CY, Wang Y, Luo GY, et al. Relationship between PD-L1 expression and CD8+ T-cell immune responses in hepatocellular carcinoma. $J$ Immunother. 2017;40(9):323-333. doi:10.1097/ CJI.0000000000000187

4. Li JH, Ma WJ, Wang GG, et al. Clinicopathologic significance and prognostic value of programmed cell death ligand 1 (PD-L1) in patients with hepatocellular carcinoma: a meta-analysis. Front Immunol. 2018;9:2077. doi:10.3389/fimmu.2018.02077

5. Conggang H, Guojun T, Jianjun W, et al. Correlation analysis between tumor metastasis and $\mathrm{T}$ cell subsets and their cytokines in primary liver cancer patients. Chongqing Med. 2017;46 (16):2215-2217.

6. Siegel RL, Miller KD, Jemal A, et al. Cancer statistics, 2017. CA: Cancer J Clin. 2017;67(1):7-30. doi:10.3322/caac.21387

7. Rasool M, Rashid S, Arooj M, et al. New possibilities in hepatocellular carcinoma treatment. Anticancer Res. 2014;34(4):1563-1571.

8. Xueman C, Erwei S. The role of tumor microenvironment in cancer immunotherapy. Adv Biochem Biophys. 2017;044(008):641-648.

9. Schreiber RD, Old LJ, Smyth MJ. Cancer immunoediting: integrating immunity's roles in cancer suppression and promotion. Science. 2011;331(6024):1565-1570. doi:10.1126/science. 1203486

10. Chung HW, Lim JB. Role of the tumor microenvironment in the pathogenesis of gastric carcinoma. World $J$ Gastroenterol. 2014;20 (07):1667-1680. doi:10.3748/wjg.v20.i7.1667 
11. Jiong S, Qiongzhu D, Xiu CL, et al. Phenotype and distribution of infiltrating lymphocytes in hepatocellular carcinoma. Chin Cancer Clin. 2015;42(11):559-563.

12. Kobayashi N, Hiraoka N, Yamagami W, et al. FOXP3+ regulatory $\mathrm{T}$ cells affect the development and progression of hepatocarcinogenesis. Clin Cancer Res. 2007;13(3):902-911. doi:10.1158/1078-0432.CCR-06-2363

13. An JL, Ji QH, An JJ, et al. Clinicopathological analysis of CD8positive lymphocytes in the tumor parenchyma and stroma of hepatocellular carcinoma. Oncol Lett. 2014;8(5):2284-2290. doi:10.3892/ ol.2014.2516

14. Xu X, Tan Y, Qian Y, et al. Clinicopathologic and prognostic significance of tumor-infiltrating CD8+ T cells in patients with hepatocellular carcinoma. Medicine. 2019;98(2).
15. Herbst RS, Soria JC, Kowanetz M, et al. Predictive correlates of response to the anti-PD-L1 antibody MPDL3280A in cancer patients. Nature. 2014;515:563. doi:10.1038/nature14011

16. Patel SP, Kurzrock R. PD-L1 expression as a predictive biomarker in cancer immunotherapy. Mol Cancer Ther. 2015;14(4):847-856. doi:10.1158/1535-7163.MCT-14-0983

17. Anwanwan D, Singh SK, Singh S, et al. Challenges in liver cancer and possible treatment approaches. Biochimica et Biophysica Acta (BBA). 2019;188314.

\section{Publish your work in this journal}

OncoTargets and Therapy is an international, peer-reviewed, open access journal focusing on the pathological basis of all cancers, potential targets for therapy and treatment protocols employed to improve the management of cancer patients. The journal also focuses on the impact of management programs and new therapeutic

Submit your manuscript here: https://www.dovepress.com/oncotargets-and-therapy-journa agents and protocols on patient perspectives such as quality of life, adherence and satisfaction. The manuscript management system is completely online and includes a very quick and fair peer-review system, which is all easy to use. Visit http://www.dovepress.com/ testimonials.php to read real quotes from published authors. 